\title{
Delayed neurological deterioration with an unknown cause subsequent to surgery for intraspinal meningiomas
}

\author{
TAO YANG, LIANG WU, XIAOFENG DENG, CHENLONG YANG, \\ YAN ZHANG, DONG ZHANG and YULUN XU
}

\begin{abstract}
Department of Neurosurgery, China National Clinical Research Center for Neurological Diseases, Beijing Tiantan Hospital, Capital Medical University, Beijing 100050, P.R. China
\end{abstract}

Received April 19, 2014; Accepted February 10, 2015

DOI: $10.3892 / \mathrm{ol} .2015 .3024$

\begin{abstract}
Delayed neurological deterioration in the absence of direct cord insult following surgical decompression is rare, but severe post-operative complication occurs in chronically compressive spinal disorders. In the present study, the clinical medical records and radiological findings of 10 patients who underwent surgical removal of intraspinal meningiomas and then experienced delayed post-operative neurological deterioration were reviewed. The cases are presented with consideration of the possible underlying mechanisms. There were five male and five female patients, with a mean age of 46.8 years. The mean duration of illness from the onset of symptoms to diagnosis was 42.8 months. Seven tumors were located in the thoracic region and three in the cervical region of the spine. The tumors compressed the cord severely and gross total removal was achieved in all cases. Immediately subsequent to the surgery, all patients were able to move all extremities, but the onset of the neurological deterioration occurred at post-operative hours 3-8 in all cases (mean, $5 \mathrm{~h}$ post-surgery). In four cases, radiological examination revealed an area of high signal changes intrinsic to the cord on T2-weighted images, but without residual compression. The mean follow-up period was 49.6 months. Nine patients reported a marked recovery in status compared with the pre-operative presentation during the several weeks to months following surgery. The surgical removal of intraspinal meningiomas may lead to delayed and severe neurological deterioration in the post-operative period in the absence of direct mechanical cord insult. Ischemia-reperfusion injury may be one potential etiology of this deterioration. Recognition of the neurological deficit following surgical excision of intraspinal meningiomas may improve pre-operative patient
\end{abstract}

Correspondence to: Professor Yulun Xu, Department of Neurosurgery, China National Clinical Research Center for Neurological Diseases, Beijing Tiantan Hospital, Capital Medical University, 6 Tiantan Xili, Beijing 100050, P.R. China

E-mail:xuhuxi@sina.cn

Key words: intraspinal meningioma, neurological deterioration, surgical removal, postoperative complication counseling and merits further study for the determination of the precise pathophysiology.

\section{Introduction}

Meningiomas of the spinal canal are relatively rare compared with the incidence in the intracranial compartment, accounting for $\sim 1.2 \%$ of all meningiomas of the central nervous system $(1,2)$. Spinal meningiomas are, in general, intradural and extramedullary. Chronic compression of the spinal cord is the main pathological mechanism and total removal of the meningioma, for cord decompression, is the primary treatment choice $(1,3)$. With the development of modern neuroradiological techniques and standard microneurosurgical procedures, intraspinal meningiomas can be removed with low morbidity and a good outcome (1). However, the development of a delayed neurological deficit in the post-operative period occurs often in patients with chronic compressive spinal cord lesions, including cervical spondylotic myelopathy, ossification of the spinal ligament and spinal stenosis (4-8), and can be identified in a small subset of patients with intraspinal meningiomas. Neurological deterioration or even paralysis subsequent to surgery is extremely rare, but these are the most serious complications (5-7).

Post-operative neurological deficit is most often due to mechanical damage resulting from the surgical procedure and intraspinal hematoma (6). However, careful surgical techniques and intraoperative neuromonitoring can indicate any potential trauma to neural tissue during the tumor removal and decompression procedures $(1,4,5)$. In the absence of clear etiology, vascular insult and toxic mechanisms may be responsible for the neurological deterioration $(4,5)$. The current study presents 284 patients that underwent surgical intervention for a spinal meningioma at Beijing Tiantan Hospital (Capital Medical University, Beijing, China). Special consideration was focused on patients that experienced post-operative neurological deterioration, in order to identify the causes and factors.

\section{Materials and methods}

Between the 2004 and 2010, 284 patients were pathologically diagnosed with intraspinal meningiomas at the Department of Neurosurgery of Beijing Tiantan Hospital. The data associated 
with the clinical presentation, radiological imaging, treatment and follow-up outcomes were collected, with approval from the Institutional Review Board of Beijing Tiantan Hospital and written informed consent was obtained from all patients. The lesion was clearly predominant in females, with the cohort comprising 226 female patients and 58 male patients, yielding a female to male ratio of 3.9:1. The age of patients ranged between 12 and 86 years, with a mean age of 52 years. The inclusion criteria consisted of: Post-operative deterioration of motor function, due to an unknown cause, by at least one level in a standard manual muscle test with aggravation of extremity function; the appearance of a novel sensory deficit subsequent to surgery; or combined deterioration of motor and sensory function. Exclusion criteria consisted of: Extradural, atypical, World Health Organization grade II or recurred tumors; meningiomas associated with neurofibromatosis; or post-operative deterioration caused by intraspinal hematoma, incomplete cord decompression or potential iatrogenic injury, which can be indicated by post-operative magnetic resonance imaging (MRI) or intraoperative monitoring.

MRI with gadolinium-contrast enhancement was performed as a standard radiological investigation prior to and following surgical treatment. T1 and T2 weighted imaging was performed in the sagittal and axial planes to determine the spinal level, size and the dural attachment of the meningioma and its association with the spinal cord.

During the initial years of the present study, mono- or multisegmental laminectomy or hemilaminectomy was performed to access the intraspinal meningioma. However, this strategy was later altered and replaced as the standard surgical approach by osteoplastic laminotomy with subsequent reconstruction of the posterior spinal column using microplates. All patients underwent surgical resection with intraoperative neurophysiological monitoring of somatosensory and motor evoked potentials.

A modified McCormick classification (Table I) $(9,10)$ was applied to assess pre- and post-operative neurological function. Structured telephone interviews were performed to determine the post-operative follow-up status of the patients.

\section{Results}

Post-operative neurological deterioration with unknown causes occurred in 10 out of 284 patients (3.5\%) (Table II). This consisted of five men and five women, aged between 32 and 62 years at presentation (mean, 46.8 years). The duration of illness from the onset of symptoms to diagnosis ranged between 10 and 120 months (mean, 42.8 months). The thoracic region of the spinal cord was affected in seven patients, and the cervical region in three patients. All patients pre-operatively presented with various degrees of sensory, motor or sphincter dysfunctions. The pre-operative assessment revealed that three patients were at Grade I of the modified McCormick classification, followed by seven at Grade Ib.

On MRI, the tumors in all cases were found to compress the cord severely and the average invasion ratio of the spinal canal was $>70 \%$, which was measured on axial T2 weighted images. All lesions were well demarcated from the spinal cord parenchyma, which facilitated their exposure and dissection. The dural attachment of the intraspinal meningioma was
Table I. Modified McCormick classification.

\begin{tabular}{ll}
\hline Grade & \multicolumn{1}{c}{ Definition } \\
\hline I & Neurologically normal \\
& Gait normal \\
& Normal professional activity \\
& Tired after walking several kilometers \\
Ib & Running is impossible, or moderate \\
& sensorimotor deficit does not significantly \\
& affect the involved limb \\
& Moderate discomfort in professional activity \\
& Presence of sensorimotor deficit affecting \\
& the function of the involved limb \\
& Mild to moderate gait difficulty \\
II & Severe pain or dysesthetic syndrome \\
& impairs quality of life \\
& Independent function and ambulation \\
& maintained \\
& More severe neurological deficit \\
& Requires cane and/or brace for ambulation \\
or maintains significant bilateral \\
upper-extremity impairment \\
III
\end{tabular}

predominantly localized laterally or ventrolaterally in four and three patients respectively, as determined by pre-operative MRI and intraoperative exploration (Fig. 1). Laminectomy or osteoplastic laminotomy with reconstruction of the posterior spinal column was performed to access the tumor. Gross total removal was achieved in all 10 cases. The dural attachment was completely resected if the tumor was located dorsally or dorsolaterally, and duraplasty was performed with artificial dura. In ventrally located tumors, the dural attachment was not excised but extensively bipolar cauterized. No somatosensory and motor-evoked potentials were affected during the surgery.

Immediately following the surgery, all patients were able to move all extremities. The onset of the neurological deficit occurred at post-operative hours 3-8 in all cases (mean, $5 \mathrm{~h}$ post-surgery). Weakness of extremities was noted in all 10 cases, and sensory disturbances were identified in four cases. The neurological assessment revealed that six patients were classified as Grade IV, followed by four as Grade III. An MRI performed immediately subsequent to surgery demonstrated that there was no pathological enhancement of the spinal cord lesion. However, in four cases, T2-weighted imaging revealed an area of high signal changes intrinsic to the cord, without residual compression, which was considered to be consistent with spinal cord edema (Fig. 2). The steroid protocol for acute spinal cord injury was immediately performed and tapering intravenous dexamethasone was added. The mean follow-up period was 49.6 months (range, 24-96 months). During 

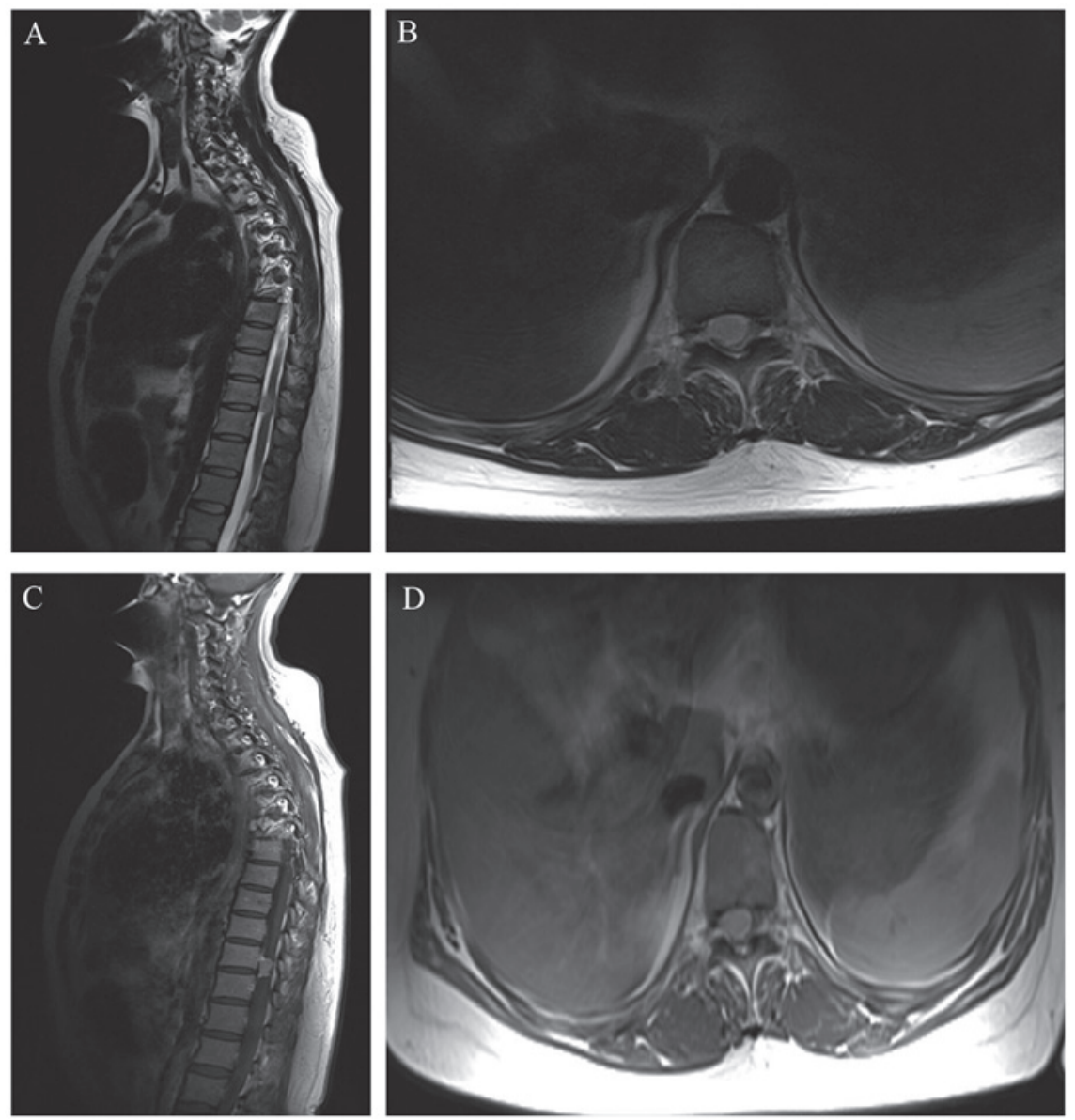

Figure 1. Pre-operative magnetic resonance imaging findings in Case 6. (A) sagittal and (B) axial T2-weighted imaging (WI) revealed a well-demarcated, mildly hyperintense extramedullary mass at the T11-12 levels of the thoracic region of the spinal cord, with marked enhancement on (C) sagittal and (D) axial T1WI with gadolinium. The tumor was located ventrolaterally within the spinal canal and severely compressed the cord.

the telephone interviews, nine patients reported a marked recovery in their status compared to the pre-operative presentation, which had occurred during the several weeks to months subsequent to surgery. The status of one patient (Case 9) exhibited little improvement at the final follow-up. At the most recent follow-up assessment, five patients had returned to Grade I, three were classified as Grade Ib, one was classified as Grade II and one was classified as Grade III on the modified McCormick scale.

\section{Discussion}

Meningiomas are common tumors in the spinal canal. Since cord compression is the main pathogenic mechanism, surgical treatment is usually successful and the outcomes are generally promising (6). Total removal of the chronic compressive spinal cord lesion in the absence of any direct trauma to the cord resulted in delayed, but severe, neurological deterioration in all 10 of the presented cases. To date, the underlying pathophysiology of such a finding remains unclear (4-8). Post-operative neurological deteriorations are usually detected on physical and neurological examination and may be a substantial disability to the patients $(4,6,8)$. There are several theories for neurological deterioration subsequent to cord decompression, and iatrogenic cord insult is a well-known possible etiology $(11,12)$. However, the timing, nature and underlying circumstances of the neurological deficits observed in the present patients suggests that alternative underlying mechanisms more likely than direct cord trauma (4-6,8). Post-operative hematomas may be a cause of neurological deterioration (13), but the patients are able to move all extremities following the surgical procedure (5,14-18). In addition, no post-operative hematomas or any compressive lesions were identified in the present study, which was consistent with previous studies $(4,5)$. Another possible etiology of post-operative paralysis subsequent to thoracic decompression is the presence of microthrombi compromising the watershed regions of arterial supply $(14,15)$. Neurapraxia during recoil in cross-sectional dimensions of the cord and a sudden drop in blood pressure also require consideration as possible etiologies for neurological deterioration (16-18).

In the present study, all tumors chronically and severely compressed the spinal cord, but the patients appeared to have compensated for the compression. It was therefore hypothesized that the acute removal and decompression of the tumors resulted in immediate cord expansion within the open canal space, and the long-term ischemic compressed segment of the cord was exposed to a rush in blood supply. This sudden cord expansion and reperfusion may have led to disruption in the blood-spinal cord barrier, and triggered a cascade of reperfusion injury, resulting in post-operative neurological deterioration. In addition, since all 10 patients experienced severe cord compression with a long duration of illness, 

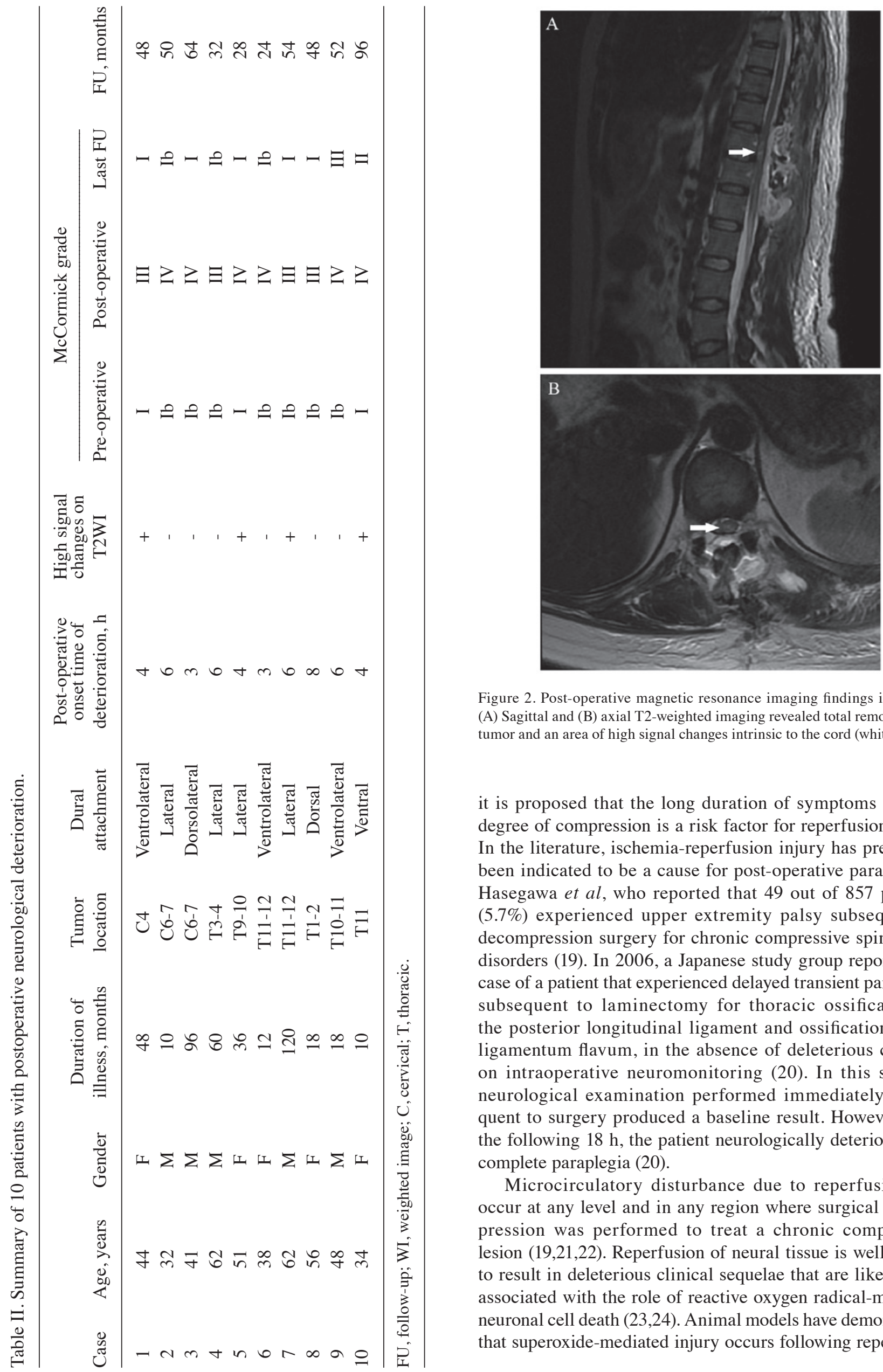

Figure 2. Post-operative magnetic resonance imaging findings in Case 6 (A) Sagittal and (B) axial T2-weighted imaging revealed total removal of the tumor and an area of high signal changes intrinsic to the cord (white arrow).

it is proposed that the long duration of symptoms and the degree of compression is a risk factor for reperfusion injury. In the literature, ischemia-reperfusion injury has previously been indicated to be a cause for post-operative paralysis by Hasegawa $e t a l$, who reported that 49 out of 857 patients (5.7\%) experienced upper extremity palsy subsequent to decompression surgery for chronic compressive spinal cord disorders (19). In 2006, a Japanese study group reported the case of a patient that experienced delayed transient paraplegia subsequent to laminectomy for thoracic ossification of the posterior longitudinal ligament and ossification of the ligamentum flavum, in the absence of deleterious changes on intraoperative neuromonitoring (20). In this study, a neurological examination performed immediately subsequent to surgery produced a baseline result. However, over the following $18 \mathrm{~h}$, the patient neurologically deteriorated to complete paraplegia (20).

Microcirculatory disturbance due to reperfusion can occur at any level and in any region where surgical decompression was performed to treat a chronic compressive lesion $(19,21,22)$. Reperfusion of neural tissue is well-known to result in deleterious clinical sequelae that are likely to be associated with the role of reactive oxygen radical-mediated neuronal cell death $(23,24)$. Animal models have demonstrated that superoxide-mediated injury occurs following reperfusion 
during acute neuronal ischemic events $(24,25)$. Furthermore, spinal cord ischemic-reperfusion injury may also appear contingent on mitochondria-dependent apoptosis, inflammatory reactions and specific phospholipid signaling cascades, resulting in neuronal injury. It has been suggested that acute and chronic spinal cord ischemic injury may induce the passage of blood-borne or neurotrophic substances, in particular TNF- $\alpha$, through the blood brain barrier (BBB) past the saturation point $(4,5,23,25)$. Decoupling of astrocyte foot processes from endothelial cell surfaces appears to inhibit tight junction function in the BBB. Transport systems and ionic buffering then become disrupted, allowing worsened reperfusion injury upon decompression of a previously ischemic spinal cord $(4,23)$. However, a potential mechanism for decompression-associated reperfusion injury of chronic ischemia has not been established at present (16-18). Substantial efforts have focused on the mitigation of spinal cord ischemic injury (4). These efforts have included surgical techniques, pharmacological interventions, and mechanical methods $(4,23,24)$. More recently, it has been suggested that potent antioxidants may play an important role in the management of spinal cord ischemic reperfusion injury $(4,5,23)$.

In four patients in the present study, post-operative MRI revealed high signal intensity on the $\mathrm{T} 2$ weighted images, which may suggest spinal cord edema. Chin et al used the term 'white cord syndrome' to describe this appearance (4). However, in certain previous studies, the increased T2-weighted signal intensity was present even prior to the decompression, and demyelination may also be a possibility for the clinical relevance, which may reflect on the various possible causes of the edema and increased signal intensity (26).

Patients and surgeons should be aware of the potentially catastrophic results of a seemingly routine tumor removal for the treatment of intraspinal meningioma with chronic but severe cord compression. It is necessary to explain the rate of neurological deterioration and possible post-surgical complications prior to operative intervention. The majority of the present patients experienced a progressive neurological deficit recovery within several weeks to months following surgery, which may aid in advising patients of the risks of spinal decompression surgery for the treatment of intraspinal meningioma.

The limitation of the present study is its retrospective nature, being performed over six years. Microneurosurgical techniques, intraoperative neuromonitoring and treatment of spinal cord injury have been considerably more developed during the follow-up period. These developments may aid in safely accomplishing tumor removal and avoiding post-operative complications, therefore exerting an effect on the surgical and clinical outcome of decompression surgery. Additionally, detailed neurological examination and surgical findings in certain early cases was not fully ascertained, since the clinical information was based on the medical records of the patients. Although 10 cases in a series appears to be a small number, the present cases constitute the first reported clinical series reporting atraumatic neurological deterioration following surgical resection for intraspinal meningiomas.

The cases presented in the current study highlight the possibility of a delayed yet severe neurological deterioration in the absence of direct insult to the spinal cord subsequent to total removal of intraspinal meningiomas. Ischemia-reperfusion injury may be one potential etiology for this deterioration. The present study may aid the improvement of the informed pre-operative decision making process and merits additional investigation into the underlying pathophysiology of the present findings.

\section{Acknowledgements}

The authors would like to thank all the patients, physicians and staff who were enrolled in and aided this study.

\section{References}

1. Sandalcioglu IE, Hunold A, Müller O, Bassiouni H, Stolke D and Asgari S: Spinal meningiomas: Critical review of 131 surgically treated patients. Eur Spine J 17: 1035-1041, 2008.

2. Setzer M, Vatter H, Marquardt G, Seifert V and Vrionis FD: Management of spinal meningiomas: Surgical results and a review of the literature. Neurosurg Focus 23: E14, 2007.

3. Ambekar S, Sharma M, Kukreja S and Nanda A: Complications and outcomes of surgery for spinal meningioma: A Nationwide Inpatient Sample analysis from 2003 to 2010. Clin Neurol Neurosurg 118: 65-68, 2014.

4. Chin KR, Seale J and Cumming V: "White cord syndrome" of acute tetraplegia after anterior cervical decompression and fusion for chronic spinal cord compression: a case report. Case Rep Orthop 2013: 697918, 2013.

5. Lee KS, Shim JJ, Doh JW, Yoon SM, Bae HG and Yun IG: Transient paraparesis after laminectomy in a patient with multi-level ossification of the spinal ligament. J Korean Med Sci 19: 624-626, 2004

6. Orchowski J, Bridwell KH and Lenke LG: Neurological deficit from a purely vascular etiology after unilateral vessel ligation during anterior thoracolumbar fusion of the spine. Spine 30 : 406-410, 2005

7. Taher F, Lebl DR, Cammisa FP, Pinter DW, Sun DY and Girardi FP: Transient neurological deficit following midthoracic decompression for severe stenosis: A series of three cases. Eur Spine J 22: 2057-2061, 2013.

8. Uematsu Y, Tokuhashi Y and Matsuzaki H: Radiculopathy after laminoplasty of the cervical spine. Spine 23: 2057-2062, 1998.

9. Aghakhani N, David P, Parker F, Lacroix C, Benoudiba F and Tadie M: Intramedullary spinal ependymomas: Analysis of a consecutive series of 82 adult cases with particular attention to patients with no preoperative neurological deficit. Neurosurgery 62: 1279-1285, discussion 1285-1286, 2008.

10. McCormick PC, Torres R, Post KD and Stein BM: Intramedullary ependymoma of the spinal cord. J Neurosurg 72: 523-532, 1990.

11. Ahn JS, Lee JK and Kim BK: Prognostic factors that affect the surgical outcome of the laminoplasty in cervical spondylotic myelopathy. Clin Orthop Surg 2: 98-104, 2010.

12. Cramer DE, Maher PC, Pettigrew DB and Kuntz C IV: Major neurologic deficit immediately after adult spinal surgery: Incidence and etiology over 10 years at a single training institution. J Spinal Disord Tech 22: 565-570, 2009.

13. Kou J, Fischgrund J, Biddinger A and Herkowitz H: Risk factors for spinal epidural hematoma after spinal surgery. Spine 27: 1670-1673, 2002.

14. Keegan JJ: The cause of dissociated motor loss in the upper extremity with cervical spondylosis. J Neurosurg 23: 528-536, 1965.

15. Sakaura H, Hosono N, Mukai Y, Ishii T and Yoshikawa H: C5 palsy after decompression surgery for cervical myelopathy: Review of the literature. Spine 28: 2447-2451, 2003.

16. Ginsburg HH, Shetter AG and Raudzens PA: Postoperative paraplegia with preserved intraoperative somatosensory evoked potentials. Case report. J Neurosurg 63: 296-300, 1985.

17. Tribus CB: Transient paraparesis: A complication of the surgical management of Scheuermann's kyphosis secondary to thoracic stenosis. Spine 26: 1086-1089, 2001.

18. Young WF and Baron E: Acute neurologic deterioration after surgical treatment for thoracic spinal stenosis. J Clin Neurosci 8: 129-132, 2001. 
19. Hasegawa K, Homma T and Chiba Y: Upper extremity palsy following cervical decompression surgery results from a transient spinal cord lesion. Spine 32: E197-E202, 2007.

20. Yamazaki M, Koda M, Okawa A and Aiba A: Transient paraparesis after laminectomy for thoracic ossification of the posterior longitudinal ligament and ossification of the ligamentum flavum. Spinal Cord 44: 130-134, 2006.

21. Chiba K, Toyama Y, Matsumoto M, Maruiwa H, Watanabe M and Hirabayashi K: Segmental motor paralysis after expansive open-door laminoplasty. Spine 27: 2108-2115, 2002.

22. Johnston CE II, Happel LT Jr, Norris R, Burke SW, King AG and Roberts JM: Delayed paraplegia complicating sublaminar segmental spinal instrumentation. J Bone Joint Surg Am 68: 556-563, 1986.

23. Shan LQ, Ma S, Qiu XC, Zhou Y, Zhang Y, Zheng LH, Ren PC, Wang YC, Fan QY and Ma BA: Hydroxysafflor Yellow A protects spinal cords from ischemia/reperfusion injury in rabbits. BMC Neurosci 11: 98, 2010.
24. Wisselink W, Money SR, Crockett DE, Nguyen JH, Becker MO, Farr GH and Hollier LH: Ischemia-reperfusion injury of the spinal cord: Protective effect of the hydroxyl radical scavenger dimethylthiourea. J Vasc Surg 20: 444-491, discussion 449-450, 1994.

25. Chan PH: Role of oxidants in ischemic brain damage. Stroke 27: 1124-1129, 1996.

26. Seichi A, Takeshita K, Kawaguchi H, Nakajima S, Akune T and Nakamura K: Postoperative expansion of intramedullary high-intensity areas on T2-weighted magnetic resonance imaging after cervical laminoplasty. Spine 29: 1478-1482, discussion 1482, 2004. 